\title{
An Introduction to Mini Black Holes at LHC
}

\author{
Horst Stöcker ${ }^{1,2}$, Ben Koch ${ }^{1,2}$, and Marcus Bleicher ${ }^{2}$ \\ ${ }^{1}$ FIAS-Frankfurt Institute for Advanced Studies, Max von Laue-Str. 1, \\ D-60438 Frankfurt am Main, Germany \\ ${ }^{2}$ Institut für Theoretische Physik, \\ Johann Wolfgang Goethe-Universität, \\ D-60438 Frankfurt am Main, Germany
}

Received on 27 February, 2007; revised version received on 3 May, 2007

\begin{abstract}
Relying on the existing estimates for the production cross sections of mini black holes in models with large extra dimensions, we review strategies for identifying those objects at collider experiments. We further consider a possible stable final state of such black holes and discuss their characteristic signatures.
\end{abstract}

Keywords: Black holes

Since the discovery of the Schwarzschild solution [1], black holes have fired mans imagination. Even more since it has been pointed out that, according to the theories with large extra dimensions (LXDs) [2] black holes (BHs) might even be produced at the Large Hadron Collider (LHC)[3]. We review possible observables of this conjecture with special emphasis on the unclear final fate of such a $\mathrm{BH}$ and the possible existence of black hole remnants (BHR) [4-15]. When doing this, we will rely on the numerous estimates of the black hole production cross section, which agree at the level of their order of magnitude [16].

\section{SOLVING THE HIERARCHY-PROBLEM WITH LARGE EXTRA DIMENSIONS}

Models which assume extra spatial dimensions can provide a solution to the so-called hierarchy problem $[2,17]$. They claim that in contrast to the Planck mass $\left(m_{P l}=1 / \sqrt{G_{N}}\right)$, which is related to Newton's gravitational constant $G_{N}$, the true fundamental scale $\left(M_{f}\right)$ might be of the order of a few $\mathrm{TeV}$. The idea of extra dimensions is of course supported by String Theory [18] but the discovery of LXDs could as little proof String Theory as a non discovery could disproof it. In our further discussion we will therefore treat the model of Arkani-Hamed, Dimopoulos and Dvali [2] as a self standing and only focus on its predictions for experiment. In this model the $d$ extra space-like dimensions are compactified on tori with radii $R$. Gravity, as the geometrical theory, sees of course the entire $(3+\mathrm{d})+1$-dimensional bulk, while all SM particles are by construction confined to our 3+1-dimensional sub-manifold (brane). This leads to a relation between $M_{f}, R$ and $m_{P l}$

$$
m_{P l}^{2}=M_{f}^{d+2} R^{d}
$$

Testing this model means finding constraints for the free parameters $d$ and $M_{f}$ see e.g. Ref. [19].

\section{BLACK HOLE PRODUCTION AND DECAY}

The Schwarzschild radius $\left(R_{H}\right)$ in $3+d$ dimensions is given by [20] $R_{H}^{d+1} \sim\left(1 / M_{f}\right)^{d+1} M / M_{f}$. In the case of compactified dimensions, this formula is applicable as long as $R_{H}$ is much smaller than the compactification radius $R$. This radius is much larger than the Schwarzschild radius corresponding to the same $\mathrm{BH}$ mass in $3+1$ dimensions. From the Hoop conjecture [21] one assumes the formation of a black hole as soon the impact parameter of two colliding particles is smaller than the corresponding Schwarzschild radius. Accordingly, this minimal impact parameter rises enormously in the extradimensional setup. The straight forward approximation of the LXD-black hole production cross section can be made by taking the classical geometric cross section

$$
\sigma(M) \approx \pi R_{H}^{2}
$$

Several aspects of this cross section have been discussed, but its order of magnitude seems to coincide in the different approaches [16]. Although we should mention here that it has recently been argued, that gravitational radiation during the formation process might carry enough energy to prevent the matter form collapsing at all [22]. The differential cross section in proton-proton collisions is then given by summation over all possible parton interactions and integration over the momentum fractions. Due to the steep behavior of the parton distribution functions [23] for small $x$, most of the black holes have masses close to the production threshold. Thus, cross sections like Eq. (2) lead to the exciting prediction that if large extra dimensions do actually exist, a large number (up to $10^{9}$ per year) of black holes will be produced in future colliders $[3,12,24-30]$ and should in fact, be daily produced in ultra high energetic cosmic ray events $[31,32]$. The order of magnitude of this prediction is very under a change of the used parton distribution function.

But what are the measurable signatures of the production of microscopical BHs in collider experiments? Most of those signatures are strongly dependent on the evaporation process [33] of the BH. This evaporation process is often classified in three phases. In the balding phase, the newly formed black hole radiates away its angular momentum by gravitational radiation. The second phase is the Hawking phase, where it is 
assumed to enter the semi-classical regime of quantum theory on the background of curved space-time. According to the Hawking law, the black hole emits radiation that is distributed by a purely thermal spectrum. The Hawking temperature is $T_{H}=M_{f}\left(M_{f} / M\right)^{1 /(d+1)}$. This relation looses its validity, as soon as the BH mass $(M)$ approaches the fundamental mass $M \approx M_{f}$. For instance it would predict that the temperature would exceed the BHs mass. This reflects the fact that the $\mathrm{BH}$ entered the regime of quantum gravity, in which no predictive theory is known and the BH's behavior and fate is unclear, so we rely on the rough, intuitive estimates of such speculative scenarios. There are two different approaches used to model the decay of a black hole numerically. One is assuming that the $\mathrm{BH}$ performs a prompt final decay into $2-6$ particles which carry the BH's charge, momentum and other quantum numbers $[34,35]$ The other is that this final decay does not take place and a remnant is left over. The idea of a remnant has been put forward to cure the information loss problem. It is supported by arguments employing the uncertainty relation [4-6], by introducing corrections to the $\mathrm{BH}$-Lagrangian $[8,9]$, by the consideration of axionic charge [10], by leading order quantum gravity considerations [11], or by quantum hair [36] arguments. These arguments are mostly made for $3+1$ dimensions, but also apply for extra dimensions.

\section{SIGNATURES FOR BLACK HOLES AND BLACK HOLE REMNANTS}

Most signatures are strongly dependent on the black hole decay. As the details of this decay are far from settled, most signatures might be artefacts on incomplete modelling. The task is to find $\mathrm{BH}$ signatures which allow conclusions on the actual physical behavior and are as model independent as possible. One of the first signatures of this kind the suppression of hadronic di-jet events above the $\mathrm{BH}$ production threshold (at $2 E_{T}>M_{f}$ ) energy [26, 28]. It relies on a Hawking radiation that will be emitted predominantly around transverse momenta of $\sim 50-500 \mathrm{GeV}$. Such radiation can not mask the standard scattering at higher $p_{T}$. With the strongly growing $\mathrm{BH}$ cross section for higher energetic collisions this results in a disappearance of high $p_{T}(>500 \mathrm{GeV})$ events in the detector. In addition to this general indicator for $\mathrm{BH}$ events the formation of stable BHRs would provide further interesting new signatures that would even allow for the identification of such a BHR event at future colliders:

- Electrically charged BHRs would leave a stiff ionizing track in the detector. This would allow to identify the BHR [15] and measure it's mass directly.

- Neutral BHRs could be identified e.g. by the $p_{t}$ distributions, multiplicities, and angular correlations $[14,15]$ of the Hawking evaporated SM particles. Here we propose a new signal for uncharged BHRs, namely the search for events with $\sim \mathrm{TeV}$ missing energy plus a quenched high $p_{T}$ hadron spectrum in the same event. Here the
BHR carries a major fraction of the total energy. While many extensions of the standard model predict missing energy signatures, here the spray of away-side hadronic Hawking-jets, above a $10 \mathrm{GeV} p_{T}$ cut off, shows a clear focusing, see Fig 1. Such events constitute, according to our simulation a significant fraction of the BHR events. An event structure like in Fig. (1) is unlikely to be produced by standard physics, as the emission of a colored particle in one direction coincides with the emission of a colored particle in the opposite direction (In the CM frame).

The very massive black holes will only contribute a marginal fraction of the total event structure, which makes the question of the BHR vs. final decay also the the dominant question for all observables that rely the final state particles.

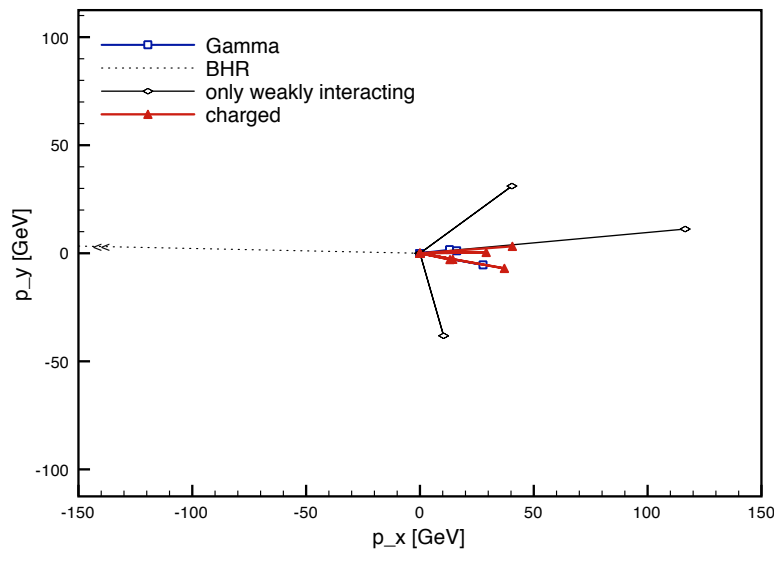

FIG. 1: Transverse momentum distribution of a single $\mathrm{BH}$ event at the LHC with an initial energy of $2 \mathrm{TeV}$ and a BHR mass of $1 \mathrm{TeV}$ and $p_{T}>10 \mathrm{GeV}$. The dashed line represents the BHR transverse momentum which, in the case of a neutral BHR, would be not visible in the detector [37].

\section{SUMMARY}

In this short report, we pointed out that most black hole observables rely on the details of the $\mathrm{BH}$ evaporation process and are therefore strongly dependent on the model that tries to simulate this process. This conceptual problem becomes even graver, as most black holes are produced close to the production threshold and therefore directly enter the final (and least understood phase) of the evaporation procedure. As a consequence we suggest to look for signatures like the suppression of hard $(\mathrm{TeV})$ di-jets above the $\mathrm{BH}$ formation threshold, which are as model independent as possible.

On this background we focus on speculations about the formation of $\mathrm{BH}$ remnants. Independent of the detailed evaporation procedure, charged stable BHRs would leave single stiff tracks in the LHC detectors, e.g. ALICE, ATLAS, and CMS. Uncharged BHRs with their very small reaction cross sections could not be observed that way. In the existing models for BHRs they could be observed by searching for 
events with $\sim 1 \mathrm{TeV}$ missing energy and quenching of the high $p_{T}$ hadron spectra.
This work was supported by GSI and $B M B F$.
[1] K. Schwarzschild, Sitzungsberichte der Deutschen Akademie der Wissenschaften zu Berlin, Klasse für Mathematik, Physik und Technik, 189 (1916)

[2] N. Arkani-Hamed, S. Dimopoulos, and G.R. Dvali, Phys. Lett. B 429, 263 (1998) hep-ph/9803315; I. Antoniadis, N. ArkaniHamed, S. Dimopoulos, and G.R. Dvali, Phys. Lett. B 436, 257 (1998) hep-ph/9804398; N. Arkani-Hamed, S. Dimopoulos, and G. R. Dvali, Phys. Rev. D 59, 086004 (1999) hep$\mathrm{ph} / 9807344$.

[3] T. Banks and W. Fischler, hep-th/9906038; Phys. Rev. Lett. 87, 161602 (2001) hep-ph/0106295; P.C. Argyres, S. Dimopoulos, and J. March-Russell, Phys. Lett. B 441, 96 (1998) hepth/9808138.

[4] M.A. Markov, in: "Proc. 2nd Seminar in Quantum Gravity", edited by M.A. Markov and P.C. West, Plenum, New York (1984).

[5] Y.B. Zel'dovich, in: "Proc. 2nd Seminar in Quantum Gravity", edited by M.A. Markov and P.C. West, Plenum, New York (1984).

[6] J.D. Barrow, E.J. Copeland, and A.R. Liddle, Phys. Rev. D 46, 645 (1992); B. Whitt, Phys. Rev. D 38, 3000 (1988).

[7] R.C. Myers and J.Z. Simon, Phys. Rev. D 38, 2434 (1988).

[8] C.G. Callan, R.C. Myers and M.J. Perry, Nucl. Phys. B 311, 673 (1988); S. Alexeyev, A. Barrau, G. Boudoul, O. Khovanskaya, and M. Sazhin, Class. Quant. Grav. 19, 4431 (2002).

[9] T.G. Rizzo, hep-ph/0503163; T.G. Rizzo, hep-ph/0504118.

[10] M.J. Bowick, S.B. Giddings, J.A. Harvey, G.T. Horowitz, and A. Strominger, Phys. Rev. Lett. 612823 (1988).

[11] A. Bonanno and M. Reuter, Phys. Rev. D 73, 083005 (2006) hep-th/0602159.

[12] S. Hossenfelder, S. Hofmann, M. Bleicher, and H. Stöcker, Phys. Rev. D 66, 101502 (2002) hep-ph/0109085; S. Hossenfelder, M. Bleicher, S. Hofmann, H. Stöcker, and A.V. Kotwal, Phys. Lett. B 566, 233 (2003) hep-ph/0302247; S. Hofmann, M. Bleicher, L. Gerland, S. Hossenfelder, K. Paech, and H. Stöcker, J. Phys. G 28, 1657 (2002); S. Hofmann, M. Bleicher, L. Gerland, S. Hossenfelder, S. Schwabe, and H. Stöcker, hep-ph/0111052; M. Bleicher, S. Hofmann, S. Hossenfelder, and H. Stöcker, Phys. Lett. B 548, 73 (2002) hep-ph/0112186; S. Hossenfelder, hep-ph/0510236; H. Stöcker, hep-ph/0605062; H. Stöcker, J. Phys. G 32, 429 (2006) hep-ph/0607165; G.L. Alberghi, R. Casadio, D. Galli, D. Gregori, A. Tronconi, and V. Vagnoni, hep-ph/0601243.

[13] S. Hossenfelder, B. Koch, and M. Bleicher, hep-ph/0507140.

[14] B. Koch, M. Bleicher, and S. Hossenfelder, JHEP 0510, 053 (2005) hep-ph/0507138.

[15] T.J. Humanic, B. Koch, and H. Stöcker, hep-ph/0607097.

[16] M.B. Voloshin, Phys. Lett. B 518, 137 (2001) hep-ph/0107119; V.S. Rychkov, hep-th/0410295; Phys. Lett. B 524, 376 (2002) hep-ph/0111099; S.B. Giddings, in Proc. of the APS/DPF/DPB Summer Study on the Future of Particle Physics (Snowmass 2001) ed. N. Graf, eConf C010630, P328 (2001) hepph/0110127; V.S. Rychkov, Phys. Rev. D 70, 044003 (2004) hep-ph/0401116; K. Kang and H. Nastase, hep-th/0409099; A. Jevicki and J. Thaler, Phys. Rev. D 66, 024041 (2002); T.G. Rizzo, in Proc. of the APS/DPF/DPB Summer Study on the Future of Particle Physics (Snowmass 2001) ed. N. Graf, eConf C010630, P339 (2001); T.G. Rizzo, hepph/0601029; D.M. Eardley and S.B. Giddings, Phys. Rev. D 66, 044011 (2002) gr-qc/0201034; T.G. Rizzo hep-ph/0606051; H. Yoshino and Y. Nambu, Phys. Rev. D 67, 024009 (2003) grqc/0209003; Phys. Lett. B 533, 153 (2002) hep-ph/0201248; D. Ida, K.Y. Oda, and S.C. Park, Phys. Rev. D 67, 064025 (2003) [Erratum-ibid. D 69, 049901 (2004)] hep-th/0212108; G.T. Horowitz and J. Polchinski, Phys. Rev. D 66, 103512 (2002) hep-th/0206228.

[17] L. Randall and R. Sundrum, Phys. Rev. Lett. 83, 4690 (1999) hep-th/9906064; Phys. Rev. Lett. 83, 3370 (1999) hep$\mathrm{ph} / 9905221$.

[18] I. Antoniadis, Phys. Lett. B 246, 377 (1990); I. Antoniadis and M. Quiros, Phys. Lett. B 392, 61 (1997) hep-th/9609209; K.R. Dienes, E. Dudas, and T. Gherghetta, Nucl. Phys. B 537, 47 (1999) hep-ph/9806292; K. R. Dienes, E. Dudas, and T. Gherghetta, Phys. Lett. B 436, 55 (1998) hep-ph/9803466.

[19] K. Cheung, hep-ph/0409028; G. Landsberg [CDF and D0 - Run II Collaboration], hep-ex/0412028.

[20] R.C. Myers and M.J. Perry, Ann. Phys. 172, 304 (1986).

[21] K.S. Thorne, in Magic without Magic, ed. J. Klauder, 231 (W.H. Freeman, San Francisco, 1972).

[22] T. Vachaspati, D. Stojkovic, and L.M. Krauss, gr-qc/0609024; T. Vachaspati and D. Stojkovic, gr-qc/0701096.

[23] The parton distribution function can be downloaded from the web page: http://www.phys.psu.edu/ cteq/

[24] R. Emparan, G.T. Horowitz, and R.C. Myers, Phys. Rev. Lett. 85, 499 (2000); P. Kanti, Int. J. Mod. Phys. A 19, 4899 (2004), V. Cardoso, M. Cavaglia, and L. Gualtieri, Phys. Rev. Lett. 96, 071301 (2006) [Erratum-ibid. 96, 219902 (2006)], V. Cardoso, M. Cavaglia, and L. Gualtieri, JHEP 0602, 021 (2006).

[25] S.B. Giddings and S. Thomas, Phys. Rev. D 65, 056010 (2002) hep-ph/0106219.

[26] K.M. Cheung, Phys. Rev. Lett. 88, 221602 (2002) hepph/0110163; Y. Uehara, hep-ph/0205068; Y. Uehara, Prog. Theor. Phys. 107, 621 (2002) hep-ph/0110382; L. Anchordoqui and H. Goldberg, Phys. Rev. D 67, 064010 (2003) hep$\mathrm{ph} / 0209337$.

[27] S. Hossenfelder, M. Bleicher, S. Hofmann, J. Ruppert, S. Scherer, and H. Stöcker, Phys. Lett. B 575, 85 (2003) hepth/0305262; R. Casadio and B. Harms, Phys. Rev. D 64, 024016 (2001) hep-th/0101154; S. Alexeyev, A. Barrau, G. Boudoul, O. Khovanskaya, and M. Sazhin, Class. Quant. Grav. 19, 4431 (2002) gr-qc/0201069; J. Alvarez-Muniz, J.L. Feng, F. Halzen, T. Han, and D. Hooper, Phys. Rev. D 65, 124015 (2002) hep-ph/0202081; I. Mocioiu, Y. Nara, and I. Sarcevic, Phys. Lett. B 557, 87 (2003) hep-ph/0301073; M. Cavaglia, S. Das, and R. Maartens, Class. Quant. Grav. 20, L205 (2003); hepph/0305223; M. Cavaglia and S. Das, Class. Quant. Grav. 21, 4511 (2004) hep-th/0404050; A. Chamblin and G. C. Nayak, Phys. Rev. D 66, 091901 (2002) hep-ph/0206060; A. Casanova and E. Spallucci, Class. Quant. Grav. 23, R45 (2006) hep$\mathrm{ph} / 0512063$.

[28] R. Casadio and B. Harms, Int. J. Mod. Phys. A 17, 4635 (2002) hep-th/0110255; I. Ya. Yref'eva, Part. Nucl. 31, 169 
(2000) hep-th/9910269; S.B. Giddings and V.S. Rychkov, Phys. Rev. D 70, 104026 (2004) hep-th/0409131; V. S. Rychkov, hep-th/0410041; T. Banks and W. Fischler, hep-th/9906038; O.V. Kancheli, hep-ph/0208021.

[29] S. Hossenfelder, Phys. Lett. B 598, 92 (2004) hep-th/0404232.

[30] D. Stojkovic, Phys. Rev. Lett. 94, 011603 (2005); D.C. Dai, G.D. Starkman, and D. Stojkovic, Phys. Rev. D 73, 104037 (2006).

[31] A. Goyal, A. Gupta, and N. Mahajan, Phys. Rev. D 63, 043003 (2001) hep-ph/0005030; R. Emparan, M. Masip, and R. Rattazzi, Phys. Rev. D 65, 064023 (2002) hep-ph/0109287; D. Kazanas and A. Nicolaidis, Gen. Rel. Grav. 35, 1117 (2003) hep-ph/0109247.

[32] A. Ringwald and H. Tu, Phys. Lett. B 525, 135 (2002) hep$\mathrm{ph} / 0111042$; J. Feng and A. Shapere, Phys. Rev. Lett. 88, 021303 (2002); A. Cafarella, C. Coriano, and T.N. Tomaras hep-ph/0410358; L.A. Anchordoqui, J.L. Feng, H. Goldberg, and A.D. Shapere, Phys. Rev. D 65, 124027 (2002) hep- ph/0112247; S.I. Dutta, M.H. Reno, and I. Sarcevic, Phys. Rev. D 66, 033002 (2002) hep-ph/0204218; D. Stojkovic and G.D. Starkman, Phys. Rev. Lett. 96, 041303 (2006); U. Harbach and M. Bleicher hep-ph/0601121; B. Koch, H.J. Drescher, and M. Bleicher, Astropart. Phys. 25, 291 (2006); astroph/0602164, E.J. Ahn, M. Ave, M. Cavaglia, and A.V. Olinto, Phys. Rev. D 68, 043004 (2003).

[33] D. Ida, K.Y. Oda, and S.C. Park, Phys. Rev. D 73, 124022 (2006) hep-th/0602188; G.L. Alberghi, R. Casadio, and A. Tronconi, hep-ph/0611009.

[34] C.M. Harris, P. Richardson, and B.R. Webber, JHEP 0308, 033 (2003) hep-ph/0307305.

[35] M. Cavaglia, R. Godang, L. Cremaldi, and D. Summers, hep$\mathrm{ph} / 0609001$.

[36] S. Coleman, J. Preskill, and F. Wilczek, Mod. Phys. Lett. A 6, 1631 (1991).

[37] B. Koch, M. Bleicher, and H. Stöcker, hep-ph/0702187. 